\title{
Semi-quantum approach for fast atom diffraction: solving the rainbow divergence
}

\author{
M.S. Gravielle ${ }^{1}$ and J.E. Miraglia ${ }^{1}$ \\ ${ }^{1}$ Instituto de Astronomía y Física del Espacio. Consejo Nacional \\ de Investigaciones Científicas y Técnicas. Casilla de Correo 67, \\ Sucursal 28, (C1428EGA) Buenos Aires, Argentina.
}

(Dated: July 23, 2014)

\begin{abstract}
In this work we introduce a distorted wave method, based on the Initial Value Representation (IVR) approach of the quantum evolution operator, in order to improve the semiclassical description of rainbow effects in diffraction patterns produced by grazing scattering of fast atoms from crystal surfaces. The proposed theory, named Surface Initial Value Representation (SIVR) approximation, is applied to He atoms colliding with a $\mathrm{LiF}(001)$ surface along low indexed crystallographic channels. For this collision system the SIVR approach provides a very good representation of the quantum interference structures of experimental projectile distributions, even in the angular region around classical rainbow angles where common semiclassical methods diverge.

PACS numbers: 34.35.+a,79.20.Rf, 34.10.+x
\end{abstract}

\section{INTRODUCTION}

The diffraction of fast atoms from crystal surfaces under grazing incidence conditions has been the focus of extensive experimental and theoretical research $1-14$ since its unexpected observation a few years ago [15, 16]. From the theoretical point of view, different methods have been employed to simulate experimental data of this phenomenon, now known as grazing-incidence fast atom diffraction (GIFAD or FAD) 17]. They range from full quantum treatments in terms of a wave packet propagation [3, 16, 18] to semiclassical approximations 1, 4] based on the use of classical projectile trajectories. Among these last theories we can mention the Surface Eikonal (SE) approximation [4, 19], which is a distorted wave method that makes use of the eikonal wave function to represent the elastic collision with the surface, while the motion of the fast projectile is classically described by considering axially channeled trajectories for different initial positions. The SE approach includes a clear description of the main mechanisms of the FAD process, being simpler to evaluate than a full quantum calculation [3, 18]. It has been applied to investigate FAD patterns for different collision systems 19 22, showing a reasonable agreement with the experiments in all the considered cases.

In spite of the successful performance of the SE approach for the simulation of FAD patterns, a weakness of the theory is its deficient description of the rainbow effect, which affects the intensity of the outermost diffraction maxima when these maxima are close to the classical rainbow angles 22], i.e. the extreme deflection angles of the classical projectile distribution . Such a deficiency, widely studied in atom-surface scattering 23], is a characteristic of the classical representation of the collision dynamics, which introduces a singularity at rainbow angles as a consequence of the presence of a point of accumulation of classical trajectories (caustics), producing cusped rainbow peaks in the angle-resolved scattering probability. In quantum mechanics, instead, these sharp rainbow peaks are replaced by smooth maxima that display an exponentially decaying behavior outside classical rainbow angles, just on the dark side, i.e. in the region of classically forbidden transitions [24]. The goal of this article is to develop a semi-quantum approximation for FAD, based on the Initial Value Representation (IVR) method by Miller [25], which can solve the drawback of the SE model without losing the simple description of the interference process in terms of classical scattering trajectories.

The IVR method represents a practical way of introducing quantum effects, such as interferences and classical forbidden processes, in classical dynamic simulations 26. Taking as starting point the Feynman path integral formulation of quantum mechanics, the basic idea of the IVR method is to introduce the standard Van Vleck approximation [27, 28] of the quantum evolution operator without considering any additional assumption. That is, within the IVR model the full quantum time evolution operator is replaced by the Van Vleck propagator in terms of classical trajectories with different initial conditions, which is evaluated numerically without using the common stationary phase approximation [26]. Precisely, this IVR strategy makes it possible to avoid the classical rainbow divergence, incorporating an approximate description of classically forbidden transitions in terms of real-valued trajectories 25]. The IVR solution has been successfully applied to different branches, providing accurate transition probabilities for several atomic, molecular and nuclear processes [25, 26, 29 33]. In most of these cases, IVR results are in excellent agreement with the corresponding full quantum values.

In this paper we extend the IVR method to deal with FAD processes by using the IVR time evolution operator in the frame of a time-dependent distorted-wave formalism. The approach proposed here, named Surface-Initial Value Representation (SIVR) approximation, is applied to evaluate FAD patterns for He atoms grazing impinging on a $\operatorname{LiF}(001)$ surface. This collision system will be used as a benchmark of the SIVR theory, comparing the 
results with available experimental data and with values derived within the SE approach.

The article is organized as follows. The theoretical formalism is derived in Sec. II. Results are presented and discussed in Sec. III, while in Sec. V we outline our conclusions. Atomic units (a.u.) are used unless otherwise stated.

\section{THEORETICAL MODEL}

When an atomic projectile $(P)$ grazingly impinges on a crystal surface $(S)$ with an incidence energy $E$, the scattering state of the projectile, $\left|\Psi_{i}^{+}(t)\right\rangle$, satisfies the time-dependent Schrödinger equation for the Hamiltonian

$$
H=-\frac{1}{2 m_{P}} \nabla_{\vec{R}}^{2}+V_{S P}(\vec{R}),
$$

where $\vec{R}$ denotes the position of the center of mass of the incident atom, $m_{P}$ is projectile mass, and $V_{S P}$ is the surface-projectile interaction. The sign " +" in the scattering state indicates that it satisfies outgoing asymptotic conditions for the elastic collision process, verifying as initial condition that at $t=0$, when the projectile is far from the surface, $\Psi_{i}^{+}$tends to the state $\Phi_{i}$, where

$$
\Phi_{j}(\vec{R}, t)=(2 \pi)^{-3 / 2} \exp \left(i \vec{K}_{j} \cdot \vec{R}-i E t\right), \quad j=i(f)
$$

is the initial (final) unperturbed wave function, with $\vec{K}_{i}$ $\left(\vec{K}_{f}\right)$ the initial (final) momentum and $E=K_{i}^{2} /\left(2 m_{P}\right)=$ $K_{f}^{2} /\left(2 m_{P}\right)$.

In the Schrödinger picture of quantum mechanics, the scattering state at a given time $t$ can be formally expressed in terms of the evolution operator $U(t)=$ $\exp (-i H t)$ as

$$
\left|\Psi_{i}^{+}(t)\right\rangle=U(t)\left|\Phi_{i}(0)\right\rangle
$$

for $t \geq 0$. A semiclassical expression of this equation can be obtained by applying the IVR method, as summarized in Ref. [26], to represent the evolution operator $U(t)$. Within the IVR approach, the scattering state of Eq. (3) becomes

$$
\begin{aligned}
\left|\Psi_{i}^{+}(t)\right\rangle \simeq & \left|\Psi_{i}^{(I V R)+}(t)\right\rangle=(2 \pi i)^{-3 / 2} \int d \vec{R}_{o} \int d \vec{K}_{o} \\
& \times\left(J_{M}(t)\right)^{1 / 2} \Phi_{i}\left(\vec{R}_{o}, 0\right) \exp \left(i S_{t}\right)\left|\overrightarrow{\mathcal{R}}_{t}\right\rangle,(4)
\end{aligned}
$$

where $\overrightarrow{\mathcal{R}}_{t} \equiv \overrightarrow{\mathcal{R}}_{t}\left(\vec{R}_{o}, \vec{K}_{o}\right)$ is the time-evolved position of the incident atom at a given time $t$, which is obtained by considering a classical trajectory with starting position and momentum $\vec{R}_{o}$ and $\vec{K}_{o}$, respectively. In Eq. (4) the function $S_{t} \equiv S_{t}\left(\vec{R}_{o}, \vec{K}_{o}\right)$ denotes the classical action along the trajectory

$$
S_{t}=\int_{0}^{t} d t^{\prime}\left[\frac{\overrightarrow{\mathcal{P}}_{t^{\prime}}^{2}}{2 m_{P}}-V_{S P}\left(\overrightarrow{\mathcal{R}}_{t^{\prime}}\right)\right]
$$

where $\overrightarrow{\mathcal{P}}_{t}$ is the classical projectile momentum at the time $t, \overrightarrow{\mathcal{P}}_{t}=m_{P} d \overrightarrow{\mathcal{R}}_{t} / d t$, while the function

$$
J_{M}(t)=\operatorname{det}\left[\frac{\partial \overrightarrow{\mathcal{R}}_{t}\left(\vec{R}_{o}, \vec{K}_{o}\right)}{\partial \vec{K}_{o}}\right]
$$

is a Jacobian factor (a determinant) evaluated along the classical trajectory $\overrightarrow{\mathcal{R}}_{t}$, which is associated with the Maslov phase. This Jacobian factor can be expressed as $J_{M}(t)=\left|J_{M}(t)\right| \exp \left(i \nu_{t} \pi\right)$, where $\left|J_{M}(t)\right|$ is the modulus of $J_{M}(t)$ and $\nu_{t}$ is an integer number that accounts for the sign of $J_{M}(t)$ at a given time $t$. In this way, $\nu_{t}$ represents a time-dependent Maslov index, satisfying that every time that $J_{M}(t)$ changes its sign along the trajectory, $\nu_{t}$ increases by 1 .

In this work we use the IVR state of Eq. (4) to describe the quantum scattering state within the framework of the time-dependent distorted-wave formalism [34]. Hence, the distorted-wave amplitude for the elastic transition from the initial state $\Phi_{i}$ to the final state $\Phi_{f}$ can be expressed as

$$
A_{i f}^{(S I V R)}=-i \int_{0}^{+\infty} d t\left\langle\Phi_{f}(t)\left|V_{S P}\right| \Psi_{i}^{(I V R)+}(t)\right\rangle .
$$

By replacing Eq. (4) in Eq. (77) and explicitly solving the integration on the spatial coordinate $\vec{R}$, which leads to a Dirac delta function in the coordinate space, the SIVR transition amplitude per unit of surface area reads

$$
A_{i f}^{(S I V R)}=\frac{1}{\mathcal{S}} \int_{\mathcal{S}} d \vec{R}_{o s} \int d \vec{K}_{o} a_{i f}^{(S I V R)}\left(\vec{R}_{o}, \vec{K}_{o}\right),
$$

where $\vec{R}_{o}=\vec{R}_{o s}+Z_{o} \widehat{z}$ is the starting position, at $t=0$, of the projectile trajectory, with $\vec{R}_{o s}$ and $Z_{o}$ the components parallel and perpendicular, respectively, to the surface plane, the $\hat{z}$ versor oriented perpendicular to the surface, aiming towards the vacuum region, and $Z_{o} \rightarrow+\infty$. In Eq. (8) the position $\vec{R}_{\text {os }}$ is integrated on a given area $\mathcal{S}$ of the surface plane, the starting momentum $\vec{K}_{o}$ satisfies the energy conservation, i.e. $\left|\vec{K}_{o}\right| \equiv K_{0}=\sqrt{2 m_{P} E}$, and

$$
\begin{aligned}
a_{i f}^{(S I V R)}\left(\vec{R}_{o}, \vec{K}_{o}\right)= & -\int_{0}^{+\infty} d t \frac{\left|J_{M}(t)\right|^{1 / 2} e^{i \nu_{t} \pi / 2}}{(2 \pi i)^{9 / 2}} V_{S P}\left(\overrightarrow{\mathcal{R}}_{t}\right) \\
& \times \exp \left[i\left(\varphi_{t}^{(S I V R)}-\vec{Q} \cdot \vec{R}_{o}\right)\right], \quad(9)
\end{aligned}
$$

is the SIVR transition amplitude associated with the classical path $\overrightarrow{\mathcal{R}}_{t} \equiv \overrightarrow{\mathcal{R}}_{t}\left(\vec{R}_{o}, \vec{K}_{o}\right)$, where $\vec{Q}=\vec{K}_{f}-\vec{K}_{i}$ is the projectile momentum transfer and $\varphi_{t}^{(S I V R)}=E t+S_{t}-\Delta_{t}$ is the SIVR phase, with $\Delta_{t}=\vec{K}_{f} \cdot\left(\overrightarrow{\mathcal{R}}_{t}-\vec{R}_{o}\right)$. 
After some steps of algebra, the SIVR phase can be expressed as

$$
\varphi_{t}^{(S I V R)}=\int_{0}^{t} d t^{\prime}\left[\frac{1}{2 m_{P}}\left(\vec{K}_{f}-\overrightarrow{\mathcal{P}}_{t^{\prime}}\right)^{2}-V_{S P}\left(\overrightarrow{\mathcal{R}}_{t^{\prime}}\right)\right],
$$

which helps to reduce numerical uncertainties due to the fact that the component of $\vec{K}_{i}$ parallel to the surface is much higher than the perpendicular one. It is interesting to note that in Eq. (9), the Jacobian factor $J_{M}(t)$ goes to zero as the Maslov index $\nu_{t}$ changes discontinuously, making the integrand be continuos at such a point [31].

The SIVR differential probability, per unit of surface area, for elastic scattering with final momentum $\vec{K}_{f}$ in the direction of the solid angle $\Omega_{f} \equiv\left(\theta_{f}, \varphi_{f}\right)$ is obtained from Eq. (8) as $d P / d \Omega_{f}=K_{f}^{2}\left|A_{i f}^{(S I V R)}\right|^{2}$, where $\theta_{f}$ and $\varphi_{f}$ are the final polar and azimuthal angles, respectively, with $\varphi_{f}$ measured with respect to the $\widehat{x}$ axis along the incidence direction in the surface plane. A schematic depiction of the process and the angular coordinates is displayed in Fig. 1.

\section{RESULTS}

We apply the SIVR method to ${ }^{4} \mathrm{He}$ atoms elastically scattered from a $\operatorname{LiF}(001)$ surface under axial surface channeling conditions. This collision system has been widely investigated with FAD [1, 4, 18 20, 35] and will be considered as a benchmark for the theory.

The SIVR transition amplitude was obtained from Eq. (8) employing the MonteCarlo technique to evaluate the $\vec{R}_{o s}$ and $\vec{K}_{o}$ integrals. The integration on $\vec{R}_{o s}$ was done using random values obtained from a Gaussian distribution, while the integral on $\vec{K}_{0}$ was solved making use of the change of variables $\vec{K}_{0}=$ $K_{0}\left(\cos \theta_{o} \cos \varphi_{o}, \cos \theta_{o} \sin \varphi_{o},-\sin \theta_{o}\right)$, with $\theta_{o}$ and $\varphi_{o}$ varying uniformly around the incidence direction, in a range determined from the uncertainty principle. That is, the $\theta_{o}$ and $\varphi_{o}$ variables were integrated in the angular ranges $\Delta \theta_{o} \simeq \pm 10\left(K_{i s} d_{z}\right)^{-1}$ and $\Delta \varphi_{o} \simeq \pm 5\left(K_{i s} d_{y}\right)^{-1}$, respectively, around the incidence direction, where $K_{i s}=$ $K_{i} \cos \theta_{i}$ is the initial momentum parallel to the surface, $\theta_{i}$ is the glancing incidence angle, and $d_{y}$ and $d_{z}$ are the lattice parameters in the directions $\widehat{y}$ and $\widehat{z}$, respectively, both of them perpendicular to the incidence channel $(\widehat{x}$ axis). More than $4 \times 10^{5}$ values of $\vec{R}_{o s}$ and $\vec{K}_{o}$ were used in the calculation of $A_{i f}^{(S I V R)}$ for each incidence condition, determined by the initial momentum $\vec{K}_{i}$. It involved the sum of the $a_{i f}^{(S I V R)}$ amplitudes corresponding to different values of $\vec{R}_{o s}$ and $\vec{K}_{o}$ that lead to the same final momentum $\vec{K}_{f}$. This was done using a grid of $100 \times 100$ points for the angles $\theta_{f}$ and $\varphi_{f}$. Every transition amplitude $a_{i f}^{(S I V R)}$ was evaluated numerically along the classical trajectory $\overrightarrow{\mathcal{R}}_{t}\left(\vec{R}_{o}, \vec{K}_{o}\right)$ from Eq. (99). In such a calculation, the evaluation of the determinant $J_{M}(t)$ represents the numerical bottleneck.

A key quantity to describe the experimental FAD patterns is the potential $V_{S P}$, which is here determined from a pairwise additive hypothesis by adding individual contributions corresponding to the interaction of the projectile with the different solid ions. Within this model, successfully employed in FAD from insulator surfaces 20, 21], the surface-projectile potential takes into account the static and polarization contributions. The static potential, derived by assuming that the electronic densities of the particles remain frozen during the collision, was evaluated as the sum of the electrostatic, kinetic and exchange potentials [36]. While in previous articles [20, 21] only local electronic density contributions were considered, in this paper we incorporate no local terms to evaluate the kinetic and exchange potentials, as given by the Lee-Lee-Parr [37] and Becke [38] models, respectively. In turn, the polarization potential, due to the rearrangement of the projectile electron density induced by the presence of target ions, was derived as in Ref. [21]. In addition, in the calculation of the static and polarization contributions we have considered a surface rumpling, with a displacement distance extracted from the ab-initio calculation of Ref. [19]. Details of the surface-potential calculation will be publish elsewhere [39].

As our main interest lies in analyzing the performance of the SIVR approach to describe rainbow effects, first we focus on the mechanism of supernumerary rainbows, which is associated with the SIVR amplitude derived from Eq. (8) by considering an area $\mathcal{S}$ equal to only one reduced unit cell [1, 19, 21]. In Fig. 2 we compare SIVR projectile distributions for a reduced unit cell with experimental data from Ref. [1] for incidence along the [100] channel with two different impact energies. The SIVR spectra, as a function of the deflection angle $\Theta$ defined as $\Theta=\arctan \left(\varphi_{f} / \theta_{f}\right)$, present well defined peaks, which can be identified as supernumerary rainbow maxima [1]. The positions and relative intensities of such peaks are in quite good agreement with the experimental data, even the rainbow maximum which presents the highest intensity. In contrast with previous semiclassical calculations 1, 1, 19, 40, within the SIVR approximation the rainbow peak is described as a smoothed maximum that takes into account the decreasing intensity on the dark side of the classical rainbow angle $\Theta_{r b}$ [24]. The angle $\Theta_{r b}$ corresponds to the largest deflection suffered for projectiles moving along classical trajectories with initial momentum $\vec{K}_{i}$, so that projectile paths ending with deflections $\Theta$ larger than $\Theta_{r b}$ are classically forbidden. Notice that despite the fact that no convolution was introduced in the SIVR spectra of Fig. 2, the SIVR probability displays a smooth behavior in the whole angular range, with a gentle change of slope as a function of $\Theta$, in accord with the experimental distribution. Theoretical spectra are expected to be symmetric with respect to the 
incidence direction, which corresponds to the deflection angle $\Theta=0$, while the experimental data are affected by experimental uncertainties that partially break such a mirror symmetry.

Similar agreement between the SIVR and experimental distributions is also observed for incidence along the [110] channel, as shown in Fig. 3. For this impact direction, the position and relative intensity of the rainbow peak are properly reproduced by the SIVR approach. However, there is a slight shift in the positions of the internal maxima, which is associated with a failure of the surface-projectile interaction model for this channel. As discussed in previous articles [3, 20, 35], FAD patterns are extremely sensitive to the corrugation of the surface potential across the incidence direction. Small differences in the potential can strongly modify the positions of supernumerary rainbow maxima, particularly, the internal ones, and this effect is more evident for incidence along the [110] channel [20].

With the aim of comparing the SIVR approach with previous semiclassical theories [4, 19, 20], in Fig. 4 we display angular projectile distributions obtained with the SIVR and SE methods, both of them including the supernumerary rainbow mechanism only, that is, derived by integrating $\vec{R}_{\text {os }}$ on a reduced unit cell. In the case of the semiclassical SE approximation, to study the role of the Maslov phase in this new context, we have considered two versions: one incorporating the Maslov phase [19] and the other without it [4, 20]. Within the SE approximation, the Maslov phase represents a correction term $\phi_{M}^{(S E)}=\nu_{o} \pi / 2$ that was added to the phase of the scattering state in order to take into account the phase change suffered by the wave as it passes through a focus, with $\nu_{o}$ the Maslov index defined as in Ref. [41]. From Fig. 4 we observe that, like other semiclassical theories [1, 17, 40], both versions of the SE approach produce an abrupt increase of the probability at classical rainbow angles $\pm \Theta_{r b}$, with null probability outside this angular range, on the dark side of the rainbow angle. This deficiency is completely solved by the SIVR method, which gives rise to smooth rainbow peaks with softened decaying intensities for deflection angles larger than $\Theta_{r b}$. The most important point to remark about the SIVR method is that the numerical integration on the starting momentum $\vec{K}_{0}$, included in Eq. (8), regularizes the divergence of the transition amplitude close to $\Theta_{r b}$, in such a way that forbidden trajectories as well as the so called Airy behavior of the quantum transition amplitude are automatically taken into account [26].

Moreover, from Fig. 4 we found that the experimental positions of supernumerary rainbow maxima are well described by the SE approach without the Maslov correction term [4, 20]. But the agreement deteriorates when the Maslov phase $\phi_{M}^{(S E)}$ is added to the SE theory, turning the central minimum into a maximum, in contrast with the experiment. On the contrary, the present SIVR approximation does incorporates a similar Maslov phase
$\phi_{M}(t)=\nu_{t} \pi / 2$ as a function of time along the classical trajectory. But this phase emerges naturally, together with the factor $\left|J_{M}(t)\right|$, in the derivation of Eq. (9), becoming in fact essential to obtain proper projectile distributions within the SIVR method. Therefore, the present results seem to indicate that the incorporation of $\phi_{M}^{(S E)}$ in the SE approach is unbalanced, in a certain manner, and it would be better to disregard it.

So far we have described the mechanism of supernumerary rainbows only, but as it happens for most of the diffraction phenomena, FAD patterns have two different origins: supernumerary rainbows and Bragg diffraction 1]. Both mechanisms are included in the SIVR description and can be analyzed separately, like in the SE approach [21]. In Eq. (8) the integration region on the surface plane, $\mathcal{S}$, is in principle determined by the size of the initial wave packet of incident projectiles [42]. By considering this area as composed by $n$ identical reduced unit cells, each of them centered on a different site $\vec{X}_{s j}$ of the crystal surface, we can express the corresponding SIVR transition amplitude as

$$
A_{i f, n}^{(S I V R)}=A_{i f, 1}^{(S I V R)} S_{n}\left(\vec{Q}_{s}\right)
$$

where $A_{i f, 1}^{(S I V R)}$ is derived from Eq. (8) by evaluating the $\vec{R}_{o s}$-integral over one reduced unit cell, while the function

$$
S_{n}\left(\vec{Q}_{s}\right)=\frac{1}{n} \sum_{j=1}^{n} \exp \left[-i \vec{Q}_{s} \cdot \vec{X}_{s j}\right]
$$

takes into account the crystallographic structure of the surface, with $\vec{Q}_{s}$ the component of $\vec{Q}$ parallel to the surface plane. Each factor in Eq. (11) describes a different mechanism. The factor $A_{i f, 1}^{(S I V R)}$ is related to supernumerary rainbows and carries information on the shape of the interaction potential across the incidence channel, while the factor $S_{n}\left(\vec{Q}_{s}\right)$ is associated with the Bragg diffraction and provides information on the spacing between surface atoms only. As the component of the momentum transfer along the incidence channel is negligible, we can approximate $S_{n}\left(\vec{Q}_{s}\right) \approx S_{n}\left(Q_{\mathrm{tr}}\right)$, where $Q_{\mathrm{tr}}=K_{f} \cos \theta_{f} \sin \varphi_{f}$ is the component of the transferred momentum transversal to the incidence channel on the surface plane. For scattering along the [110] channel this function reads

$$
S_{n}^{[110]}\left(Q_{\mathrm{tr}}\right)=\frac{\sin \left(n_{\mathrm{tr}} \beta\right)}{n_{\mathrm{tr}} \sin \beta},
$$

while for incidence along the [100] channel it reads

$$
S_{n}^{[100]}\left(Q_{\mathrm{tr}}\right)=\left(n_{\mathrm{tr}}^{2}+1\right)^{-1}\left[n_{\mathrm{tr}}^{2} \frac{\sin \left(n_{\mathrm{tr}} \beta\right)}{n_{\mathrm{tr}} \sin \beta}+\frac{\cos \left(n_{\mathrm{tr}} \beta\right)}{\cos \beta}\right],
$$

where $n_{\mathrm{tr}}$ is the number of reduced unit cells along the transverse direction (fixed as an odd number) and $\beta=Q_{\operatorname{tr}} d / 2$, with $d$ the spatial lattice periodicity of the 
channel. Hence, in both directions $S_{n}\left(Q_{\mathrm{tr}}\right)$ gives rise to Bragg maxima placed at

$$
Q_{\mathrm{tr}} d=m 2 \pi,
$$

with $m$ an integer number. The width of these diffraction peaks is affected by the number of reduced unit cells reached by the incident wave packet, i.e. the larger $n_{\mathrm{tr}}$ is, the narrower the Bragg peaks are.

To visualize the above behavior, in Fig. 5 we display the SIVR distribution obtained from Eq. (8) by integrating $\vec{R}_{o S}$ on an area $\mathcal{S}$ formed by three reduced unit cells. In this case, the SIVR spectrum presents Bragg maxima as superimposed structures to the supernumerary contribution. Resolved Bragg peaks can be observed in experimental projectile distributions for low values of the perpendicular energy $E_{\perp}=E \sin ^{2} \theta_{i}$, associated with the motion normal to the surface plane [19]. But for higher perpendicular energies, like the ones considered in Figs. 2 and 3, discrete Bragg peaks originated from the interference of trajectories from different reduced unit cells are not present in the experimental distributions due to the limits in spatial resolution of the detector [21]. Therefore, only supernumerary rainbow contributions are visible in the experimental spectra of such figures.

\section{CONCLUSIONS}

We have developed a semi-quantum approximation based on the IVR method of Miller's [25] to deal with FAD from crystal surfaces. The proposed approach the SIVR approximation - solves the rainbow singular- ities originated by the classical description of the projectile dynamics, preserving a simple semi-quantum picture of the main mechanisms of the process. In order to test the reliability of the SIVR method, we have applied it to keV He atoms colliding under grazing incidence with a $\operatorname{LiF}(001)$ surface, for which there are available experimental data. The surface potential was derived from a pairwise additive model, including non local kinetics and exchange contributions, polarization and rumpling. From the comparison of calculated angular spectra with experimental projectile distributions for two different low-indexed crystallographic directions of the LiF surface we conclude that the SIVR approach provides a very good representation of the FAD patterns in the whole angular range, without requiring the use of convolutions to smooth the theoretical curves. Therefore, the SIVR method might be considered as an attractive alternative to quantum wave packet propagations, offering a realistic description of FAD patterns, even around classical rainbow angles.

We also found that the use of the Maslov correction term in the SE approximation might be inadequate, while in the SIVR approximation the Maslov phase emerges as a function of the projectile position along the classical trajectory, playing an essential role.

\section{Acknowledgments}

M.S.G. is kindly grateful to Marcos Saraceno for his helpful suggestion. M.S.G and J.E.M acknowledge financial support from CONICET, UBA, and ANPCyT of Argentina.
[1] A. Schüller and H. Winter, Phys. Rev. Lett. 100, 097602 (2008).

[2] J.R. Manson, H. Khemliche, and P. Roncin, Phys. Rev. B 78, 155408 (2008).

[3] F. Aigner, N. Simonović, B. Solleder, L. Wirtz, and J. Burgdörfer, Phys. Rev. Lett. 101, 253201 (2008).

[4] M.S. Gravielle and J.E. Miraglia, Phys. Rev. A. 78, 022901 (2008).

[5] N. Bundaleski, H. Khemliche, P. Soulisse, and P. Roncin, Phys. Rev. Lett. 101, 177601 (2008).

[6] A. Schüller, M. Busch, J. Seifert, S. Wethekam, H. Winter, and K. Gärtner, Phys. Rev. B 79, 235425 (2009).

[7] A, Ruiz, J.P. Palao, and E.J. Heller, Phys. Rev. A 79, 052901 (2009).

[8] C. Díaz, P. Rivière, and F. Martín, Phys. Rev. Lett. 103, 013201 (2009).

[9] H. Khemliche, P. Rousseau, P. Roncin, V.H. Etgens, and F. Finocchi, Appl. Phys. Lett. 95, 151901 (2009).

[10] H. Winter, J. Seifert, D. Blauth, M. Busch, A. Schüller, and S. Wethekam, Appl. Surf. Sci. 256, 365 (2009).

[11] A. Schüller, M. Busch, S. Wethekam, and H. Winter, Phys. Rev. Lett. 102, 017602 (2009).

[12] J. Seifert, A. Schüller, H. Winter, R. Wlodarczyk, J.
Sauer, and M. Sierka, Phys. Rev. B 82, 035436 (2010).

[13] J. Seifert and H. Winter, Phys. Rev. Lett. 108, 065503 (2012).

[14] J. Seifert, M. Busch, E. Meyer and H. Winter, Phys. Rev. Lett. 111, 137601 (2013).

[15] A. Schüller, S. Wethekam, and H. Winter, Phys. Rev. Lett. 98, 016103 (2007).

[16] P. Rousseau, H. Khemliche, A.G. Borisov, and P. Roncin, Phys. Rev. Lett. 98, 016104 (2007).

[17] H. Winter and A. Schüller, Prog. Surf. Sci. 86, 169 (2011) and references therein.

[18] A. Zugarramurdi and A.G. Borisov, Phys. Rev. A. 86, 062903 (2012).

[19] A. Schüller, H. Winter, M.S. Gravielle, J.M Pruneda, and J.E. Miraglia, Phys Rev. A 80, 062903 (2009).

[20] M.S. Gravielle and J.E. Miraglia, Nucl. Instrum. Meth. Phys. Res. B 267, 610 (2009).

[21] M.S. Gravielle, A. Schüller, H. Winter, and J.E. Miraglia, Nucl. Instrum. Meth. Phys. Res. B 269, 1208 (2011).

[22] C.A. Ríos Rubiano, G.A. Bocan, M.S. Gravielle, N. Bundaleski, H. Khemliche, and P. Roncin, Phys. Rev. A 87, 012903 (2013).

[23] S. Miret-Artés and E. Pollak, Surf. Sci. Rep. 67, 161 


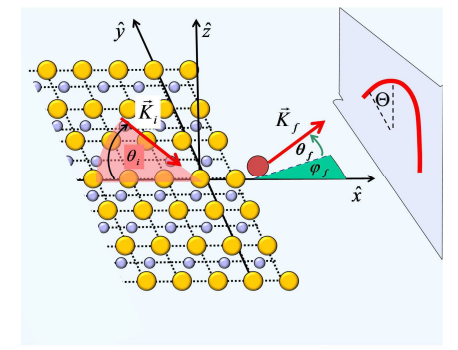

Fig. 1

FIG. 1: (Color online) Sketch of the angular coordinates for the FAD process.

(2012) and references therein.

[24] M.V. Berry and K.E. Mount, Rep. Prog. Phys. 35, 315 (1972).

[25] W.H. Miller, J. Chem. Phys. 53, 3578 (1970).

[26] W.H. Miller, J. Phys.Chem. A 105, 2942 (2001) and references therin.

[27] J.V. Van Vleck, Proc. Nat. Acad. Sci. USA 14, 178 (1928).
[28] D.J. Tannor, Introduction to Quantum Mechanics: A time-dependent perspective (University Science Books, Sausalito, 2007), chap. 10.

[29] X. Sun and W.H. Miller, J. Chem. Phys. 106, 916 (1997).

[30] X. Sun and W.H. Miller, J. Chem. Phys. 106, 6346 (1997).

[31] X. Sun and W.H. Miller, J. Chem. Phys. 108, 8870 (1998).

[32] D.E. Skinner and W.H. Miller, Chem. Phys. Lett. 300, 20 (1999).

[33] C.H. Dasso, M.I. Gallardo, and M. Saraceno, Phys. Rev. C 75, 054611 (2007).

[34] D.P. Dewangan and J. Eichler, Phys. Rep. 247, 59 (1994).

[35] A. Schüller et al., Phys. Rev. A 82, 062902 (2010).

[36] Y.S. Kim and R.G. Gordon, J. Chem. Phys. 60, 4323 (1974).

[37] H. Lee, C. Lee, and R.G. Parr, Phys. Rev. A 44, 768 (1991).

[38] A.D. Becke, Phys. Rev. A 38, 3098 (1988).

[39] J.E. Miraglia and M.S. Gravielle, to be published.

[40] A. Schüller et al., Surf. Sci. 606, 161 (2012).

[41] W.F. Avrin and R.P. Merrill, Surf. Sci. 311, 269 (1994).

[42] C.J. Joachain, Quantum Collision Theory (NorthHolland, Amsterdam, 1979). 


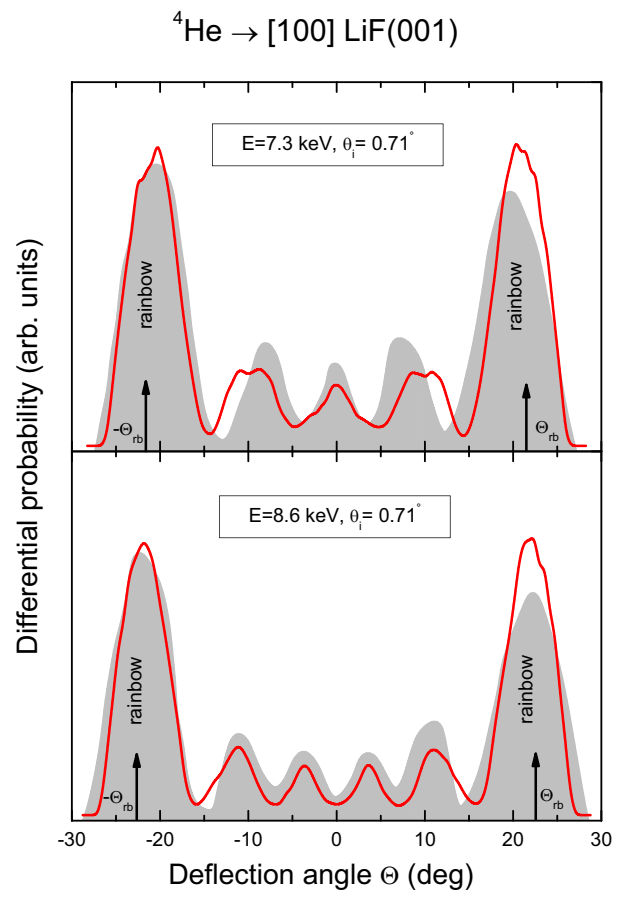

Fig. 2

FIG. 2: (Color online) Angular projectile distribution, as a function of the deflection angle $\Theta$, for ${ }^{4} \mathrm{He}$ atoms scattered from $\mathrm{LiF}(001)$ along the [100] direction with the glancing incidence angle $\theta_{i}=0.71 \mathrm{deg}$. Two different impact energies are considered: (a) $E=7.3 \mathrm{keV}$; (b) $E=8.6 \mathrm{keV}$. Solid red line, SIVR results for the supernumerary rainbow mechanism, derived by integrating the starting position $\vec{R}_{o s}$ over a reduced unit cell; shadow gray line, experimental data from Ref. [1]. Vertical arrows, positions of the classical rainbow angles $\pm \Theta_{r b}$.

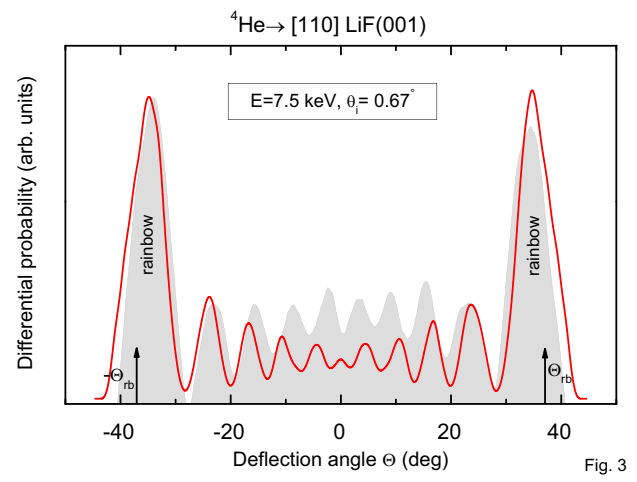

FIG. 3: (Color online) Similar to Fig. 2 for ${ }^{4} \mathrm{He}$ atoms scattered from $\operatorname{LiF}(001)$ along the [110] channel. The incidence energy and angle are $E=7.5 \mathrm{keV}$ and $\theta_{i}=0.67$ deg., respectively. The experimental data were extracted from Ref. [19]. 


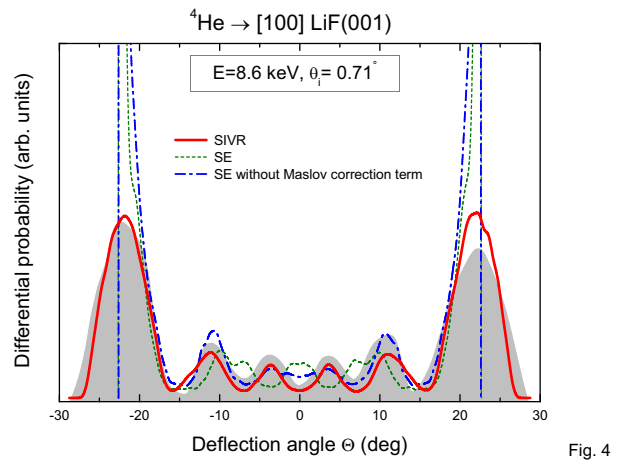

FIG. 4: (Color online) Similar to Fig. 2 (b) considering different theoretical descriptions of the supernumerary rainbow mechanism. Solid red line, SIVR approximation; dashed green line, SE approach; dash-dotted blue line, SE approach without including the Maslov correction term, as explained in the text. All the theories evaluated integrating the starting position $\vec{R}_{o s}$ over a reduced unit cell.

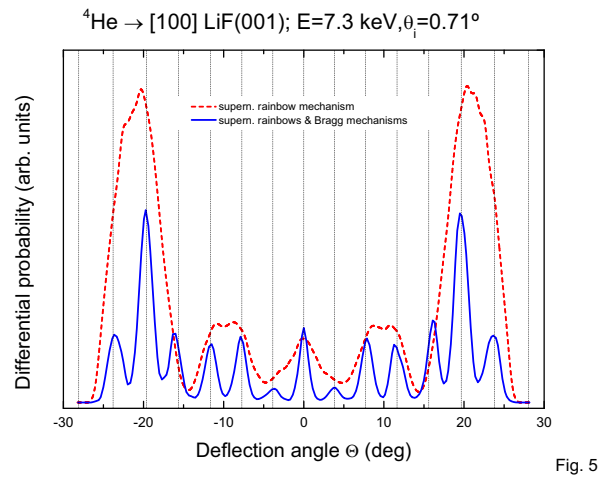

FIG. 5: (Color online) Similar to Fig. 2 (a) comparing the contributions of the different mechanisms. Dashed red line, SIVR results derived from Eq. (8) by integrating the starting position $\vec{R}_{\text {os }}$ over a reduced unit cell (supernumerary rainbow contribution); solid blue line, similar by using an extended integration area, as explained in the text. Dotted vertical lines, theoretical peak positions based on the Bragg condition (Eq. (15)). 